\title{
Vacuum stability with the effective six-pointed couplings of the Higgs bosons in the heavy supersymmetry
}

\author{
Mikhail Dubinin ${ }^{1,2, \star}$ and Elena Petrova ${ }^{1,2, \star \star}$ \\ ${ }^{1}$ Skobeltsyn Institute of Nuclear Physics, Lomonosov Moscow State University, 119991 Moscow, Russia \\ ${ }^{2}$ Physics Department, Lomonosov Moscow State University, 119991 Moscow, Russia
}

\begin{abstract}
Consistent and phenomenologically successful scenarios of the Minimal Supersymmetric Standard Model (MSSM) include the Higgs potential which is positively defined at large fields and has two minima, one of them is true and stable vacuum in which we live. However, the effective terms of the MSSM potential which appear at a loop level can spoil the vacuum stability leading to a metastable or unstable vacuum configurations. Such a situation is analysed and the general necessary conditions of vacuum stability in the MSSM parameter space are imposed. For a majority of the MSSM "benchmark scenarios" which are used for reconstruction of production channels at the LHC, the effective potential is found to be always stable, except light stop and $\tau$-phobic scenarios.
\end{abstract}

\section{Introduction}

The discovery of the Higgs boson with mass $125.09 \pm 0.24 \mathrm{GeV}$ at the LHC [1] confirmed the fundamental concept of symmetry breaking in the scalar sector. The properties of a new scalar, e.g. its spin, $\mathrm{CP}$ parity, and coupling strengths to the SM particles, are consistent within the statistical uncertainties with predictions of the Standard Model (SM). However, statistical uncertainties of the signal strength and significant signal strength errors in the production channels are not small and should be reduced by the future analyses of ATLAS and CMS Collaborations at the energy $13 \mathrm{TeV}$. Combined results [2] for the Higgs boson production and decay rates as well as constraints on its couplings to the SM particles leave a room for meaningful contributions of physics beyond the SM.

Precise measurements of the Higgs mass and the top quark mass are critical to determine the vacuum structure of the SM [3] which demonstrates a metastable vacuum. In the SM the scalar sector is single-field in the unitary gauge. In the SM extensions, the situation is more complicated because the Higgs sector includes several fields.

\section{The MSSM effective Higgs potential at the one-loop}

In the MSSM the Higgs sector consists of five Higgs bosons. In the CP-conserving limit, they are two $C P$-even neutral Higgs bosons $h$ and $H$, one $C P$-odd neutral Higgs boson $A$ and two charged scalars

\footnotetext{
${ }^{\star}$ e-mail: dubinin@ theory.sinp.msu.ru

$\star \star$ e-mail: petrova@theory.sinp.msu.ru
} 
$H^{ \pm}$[4]. As a rule, the lightest CP-even scalar $h$ is identified with the observable Higgs boson. Large radiative corrections at loop level ensure the $h$-boson mass of approximately $125 \mathrm{GeV}$. The effective Higgs potential in the Coleman-Weinberg framework [5] expanded to all orders of perturbation theory is shown schematically in figure 1 and has a form

$$
U(1-\text { loop })=U^{(2)}+U^{(4)}+U^{(6)}+\ldots
$$

In the literature, it is usually supposed that all higher order potential terms of effective operators more

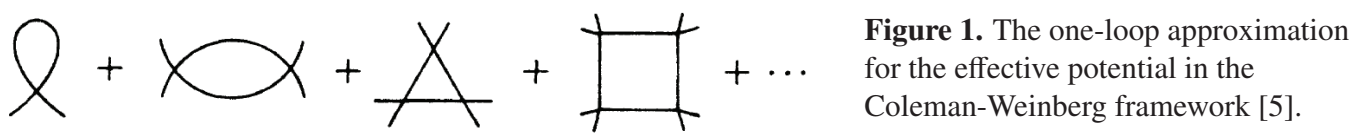

then dimension-four in the fields are negligibly small if the following conditions are respected [6]

$$
2\left|m_{t} A_{t}\right|<M_{S}^{2}, \quad 2\left|m_{t} \mu\right|<M_{S}^{2},
$$

where $m_{t}=173.2 \mathrm{GeV}$ is the top quark mass, $A_{t}$ is the trilinear Higgs- $t$-squark interaction parameter, $\mu$ is the Higgs superfield mass parameter and $M_{S}$ is the squark mass scale. This parametrization of the MSSM soft supersymmetry breaking sector [7] is the most common in the MSSM benchmark scenarios [8]. However, the dimension-six operators may play an important role in the $A_{t}, \mu$ parameter range about/of the order of $10^{1} \mathrm{TeV}$ and low and moderate $M_{S}$ [9]. Such a situation is rather unusual in the most of MSSM scenarios but in general parameters $A_{t}, \mu$ may have so large values which respect the perturbative unitarity conditions (see e.g. [10]). Potential terms of the two-doublet scalar sector can be written as follows

$$
\begin{aligned}
U^{(2)} & =-\mu_{1}^{2}\left(\Phi_{1}^{\dagger} \Phi_{1}\right)-\mu_{2}^{2}\left(\Phi_{2}^{\dagger} \Phi_{2}\right)-\left[\mu_{12}^{2}\left(\Phi_{1}^{\dagger} \Phi_{2}\right)+h . c .\right], \\
U^{(4)} & =\lambda_{1}\left(\Phi_{1}^{\dagger} \Phi_{1}\right)^{2}+\lambda_{2}\left(\Phi_{2}^{\dagger} \Phi_{2}\right)^{2}+\lambda_{3}\left(\Phi_{1}^{\dagger} \Phi_{1}\right)\left(\Phi_{2}^{\dagger} \Phi_{2}\right)+\lambda_{4}\left(\Phi_{1}^{\dagger} \Phi_{2}\right)\left(\Phi_{2}^{\dagger} \Phi_{1}\right)+ \\
& +\left[\lambda_{5} / 2\left(\Phi_{1}^{\dagger} \Phi_{2}\right)\left(\Phi_{1}^{\dagger} \Phi_{2}\right)+\lambda_{6}\left(\Phi_{1}^{\dagger} \Phi_{1}\right)\left(\Phi_{1}^{\dagger} \Phi_{2}\right)+\lambda_{7}\left(\Phi_{2}^{\dagger} \Phi_{2}\right)\left(\Phi_{1}^{\dagger} \Phi_{2}\right)+\text { h.c. }\right], \\
U^{(6)} & =\kappa_{1}\left(\Phi_{1}^{\dagger} \Phi_{1}\right)^{3}+\kappa_{2}\left(\Phi_{2}^{\dagger} \Phi_{2}\right)^{3}+\kappa_{3}\left(\Phi_{1}^{\dagger} \Phi_{1}\right)^{2}\left(\Phi_{2}^{\dagger} \Phi_{2}\right)+\kappa_{4}\left(\Phi_{1}^{\dagger} \Phi_{1}\right)\left(\Phi_{2}^{\dagger} \Phi_{2}\right)^{2}+ \\
& +\kappa_{5}\left(\Phi_{1}^{\dagger} \Phi_{1}\right)\left(\Phi_{1}^{\dagger} \Phi_{2}\right)\left(\Phi_{2}^{\dagger} \Phi_{1}\right)+\kappa_{6}\left(\Phi_{1}^{\dagger} \Phi_{2}\right)\left(\Phi_{2}^{\dagger} \Phi_{1}\right)\left(\Phi_{2}^{\dagger} \Phi_{2}\right)+ \\
& +\left[\kappa_{7}\left(\Phi_{1}^{\dagger} \Phi_{2}\right)^{3}+\kappa_{8}\left(\Phi_{1}^{\dagger} \Phi_{1}\right)^{2}\left(\Phi_{1}^{\dagger} \Phi_{2}\right)+\kappa_{9}\left(\Phi_{1}^{\dagger} \Phi_{1}\right)\left(\Phi_{1}^{\dagger} \Phi_{2}\right)^{2}+\right. \\
& +\kappa_{10}\left(\Phi_{1}^{\dagger} \Phi_{2}\right)^{2}\left(\Phi_{2}^{\dagger} \Phi_{2}\right)+\kappa_{11}\left(\Phi_{1}^{\dagger} \Phi_{2}\right)^{2}\left(\Phi_{2}^{\dagger} \Phi_{1}\right)+\kappa_{12}\left(\Phi_{1}^{\dagger} \Phi_{2}\right)\left(\Phi_{2}^{\dagger} \Phi_{2}\right)^{2}+ \\
& \left.+\kappa_{13}\left(\Phi_{1}^{\dagger} \Phi_{1}\right)\left(\Phi_{1}^{\dagger} \Phi_{2}\right)\left(\Phi_{2}^{\dagger} \Phi_{2}\right)+h . c .\right],
\end{aligned}
$$

where

$$
\Phi_{i}=\left(\begin{array}{c}
\phi_{i}^{+}(x) \\
\phi_{i}^{0}(x)
\end{array}\right)=\left(\begin{array}{c}
-i \omega_{i}^{+} \\
\frac{1}{\sqrt{2}}\left(v_{i}+\eta_{i}+i \chi_{i}\right)
\end{array}\right), \quad i=1,2
$$

- Higgs doublets with the $S U(2)$ field states and $v_{1}=v \cos \beta, v_{2}=v \sin \beta(v=246 \mathrm{GeV})$ - vacuum expectation values of them. The real part of $\mu_{12}^{2}$ is fixed by zero eigenvalue of the mass matrix (which ensures massless Goldstone boson state and defines the $C P$-odd scalar mass $m_{A}^{2}$, see details in ref. [9])

$$
\begin{aligned}
\operatorname{Re} \mu_{12}^{2} & =s_{\beta} c_{\beta}\left[m_{A}^{2}+\frac{v^{2}}{2}\left(2 \operatorname{Re} \lambda_{5}+\operatorname{Re} \lambda_{6} \cot \beta+\operatorname{Re} \lambda_{7} \tan \beta\right)\right]+v^{4}\left\{\operatorname{Re} \kappa_{9} c_{\beta}^{3} s_{\beta}\right. \\
& \left.+\operatorname{Re} \kappa_{10} c_{\beta} s_{\beta}^{3}+\frac{1}{4}\left[\operatorname{Re} \kappa_{8} c_{\beta}^{4}+\operatorname{Re} \kappa_{12} s_{\beta}^{4}+\left(9 \operatorname{Re} \kappa_{7}+\operatorname{Re} \kappa_{11}+\operatorname{Re} \kappa_{13}\right) s_{\beta}^{2} c_{\beta}^{2}\right]\right\}
\end{aligned}
$$


where $s_{\beta}=\sin \beta, c_{\beta}=\cos \beta$ and so on. Tree level boundary conditions for Higgs self-couplings at the scale $M_{S}$ are [11]

$$
\begin{aligned}
\lambda_{1,2}^{\text {tree }}\left(M_{S}\right)= & \frac{g_{1}^{2}+g_{2}^{2}}{4}, \quad \lambda_{3}^{\text {tree }}\left(M_{S}\right)=\frac{g_{2}^{2}-g_{1}^{2}}{4}, \quad \lambda_{4}^{\text {tree }}\left(M_{S}\right)=\frac{g_{2}^{2}}{2}, \\
& \lambda_{5,6,7}^{\text {tree }}\left(M_{S}\right)=0, \quad \kappa_{1, \ldots, 13}^{\text {tree }}\left(M_{S}\right)=0,
\end{aligned}
$$

where $g_{1}$ and $g_{2}$ are the standard gauge couplings, while at the loop-level and below $M_{S}$ scale these couplings acquire radiative corrections

$$
\begin{gathered}
\lambda_{1,2}(M)=\lambda_{1,2}^{\text {tree }}\left(M_{S}\right)-\Delta \lambda_{1,2}(M) / 2, \quad \lambda_{3, \ldots, 7}(M)=\lambda_{3, \ldots, 7}^{\text {tree }}\left(M_{S}\right)-\Delta \lambda_{3, \ldots, 7}(M), \\
\kappa_{1, \ldots 13}(M)=\Delta \kappa_{1, \ldots 13}(M) .
\end{gathered}
$$

Radiative corrections to parameters $\lambda_{i}(i=1, \ldots 7)$ in the effective field theory framework have been analysed in ref. $[6,7,12,13]$. Radiative corrections to the parameters $\kappa_{i}(i=1, \ldots 13)$ in the approximation of degenerate squark masses have been obtained in ref. [9]. An example of the one-loop renormalization group improved (RG-improved) threshold correction for $\lambda_{1}$ and the threshold correction for $\kappa_{1}$ can be written as

$$
\begin{aligned}
-\frac{\Delta \lambda_{1}^{\mathrm{thr}}}{2} & =\frac{3}{32 \pi^{2}}\left[h_{b}^{4} \frac{\left|A_{b}\right|^{2}}{M_{S}^{2}}\left(2-\frac{\left|A_{b}\right|^{2}}{6 M_{S}^{2}}\right)-h_{t}^{4} \frac{|\mu|^{4}}{6 M_{S}^{4}}+2 h_{b}^{4} l+\frac{g_{2}^{2}+g_{1}^{2}}{4 M_{S}^{2}}\left(h_{t}^{2}|\mu|^{2}-h_{b}^{2}\left|A_{b}\right|^{2}\right)\right] \\
& +\frac{1}{768 \pi^{2}}\left(11 g_{1}^{4}+9 g_{2}^{4}-36\left(g_{1}^{2}+g_{2}^{2}\right) h_{b}^{2}\right) l \\
\Delta \kappa_{1}^{\mathrm{thr}} & =\frac{h_{b}^{6}}{32 M_{S}^{2} \pi^{2}}\left(2-\frac{3\left|A_{b}\right|^{2}}{M_{S}^{2}}+\frac{\left|A_{b}\right|^{4}}{M_{S}^{4}}-\frac{\left|A_{b}\right|^{6}}{10 M_{S}^{6}}\right)-h_{b}^{4} \frac{g_{1}^{2}+g_{2}^{2}}{128 M_{S}^{2} \pi^{2}}\left(3-3 \frac{\left|A_{b}\right|^{2}}{M_{S}^{2}}+\frac{\left|A_{b}\right|^{4}}{2 M_{S}^{4}}\right) \\
& +\frac{h_{b}^{2}}{512 M_{S}^{2} \pi^{2}}\left(\frac{5}{3} g_{1}^{4}+2 g_{1}^{2} g_{2}^{2}+3 g_{2}^{4}\right)\left(1-\frac{\left|A_{b}\right|^{2}}{2 M_{S}^{2}}\right)-h_{t}^{6} \frac{|\mu|^{6}}{320 M_{S}^{8} \pi^{2}} \\
& +h_{t}^{4} \frac{\left(g_{1}^{2}+g_{2}^{2}\right)|\mu|^{4}}{256 M_{S}^{6} \pi^{2}}-h_{t}^{2} \frac{\left(17 g_{1}^{4}-6 g_{1}^{2} g_{2}^{2}+9 g_{2}^{4}\right)|\mu|^{2}}{3072 M_{S}^{4} \pi^{2}}+\frac{g_{1}^{2}}{1024 M_{S}^{2} \pi^{2}}\left(g_{1}^{4}-g_{2}^{4}\right)
\end{aligned}
$$

where $l \equiv \ln \left(\frac{M_{S}^{2}}{\sigma^{2}}\right), \sigma$ is the renormalization scale, $h_{t}=\frac{g_{2} m_{t}}{\sqrt{2} m_{W} \sin \beta}$ and $h_{b}=\frac{g_{2} m_{b}}{\sqrt{2} m_{W} \cos \beta}$ are Yukawa couplings. One can notice inspecting these explicit forms that radiative corrections $\Delta \kappa^{\text {thr }}$ begin to play an important role if the following conditions are true

$$
\begin{gathered}
|\mu| m_{t} \cot \beta \approx M_{S}^{2}, \quad|\mu| m_{b} \tan \beta \approx M_{S}^{2}, \quad\left|A_{t}\right| m_{t} \approx M_{S}^{2}, \quad\left|A_{b}\right| m_{b} \approx M_{S}^{2}, \\
\left|\mu A_{t}\right| m_{t}^{2} \cot \beta \approx M_{S}^{4}, \quad\left|\mu A_{b}\right| m_{b}^{2} \tan \beta \approx M_{S}^{4} .
\end{gathered}
$$

In this case, the corrections coming from the dimension-six operators of the one-loop potential can be appreciable even if the inequalities (2) are respected.

\section{Vacuum stability with respect to $\Delta \kappa_{1, \ldots 13}$ terms}

The local minimum $v=246 \mathrm{GeV}$ of the Higgs potential exists if the following conditions are respected

$$
\left.\frac{\partial U}{\partial \phi_{1}^{0}}\right|_{\phi_{i}^{0}=v_{i}}=0,\left.\quad \frac{\partial U}{\partial \phi_{2}^{0}}\right|_{\phi_{i}^{0}=v_{i}}=0, \quad \Delta=\|\left.\begin{array}{cc}
U_{\phi_{1}^{0} \phi_{1}^{0}}^{\prime \prime} & U_{\phi_{1}^{0} \phi_{2}^{0}}^{\prime \prime} \\
U_{\phi_{2}^{0} \phi_{1}^{0}}^{\prime \prime} & U_{\phi_{2}^{0} \phi_{2}^{0}}^{\prime \prime}
\end{array}\right|_{\phi_{i}^{0}=v_{i}}>0,\left.\quad U_{\phi_{1}^{0} \phi_{1}^{0}}\right|_{\phi_{i}^{0}=v_{i}}>0 .
$$




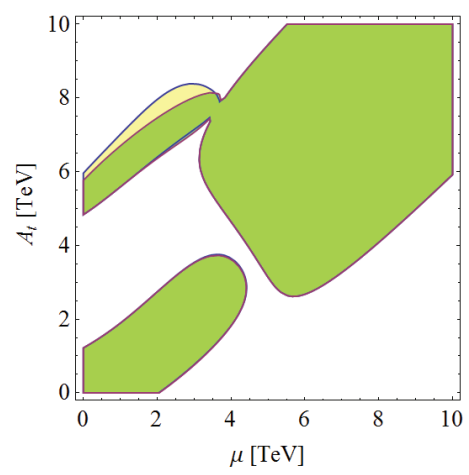

(a)

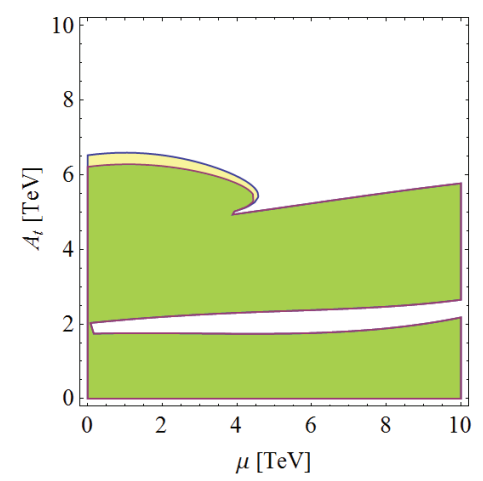

(b)

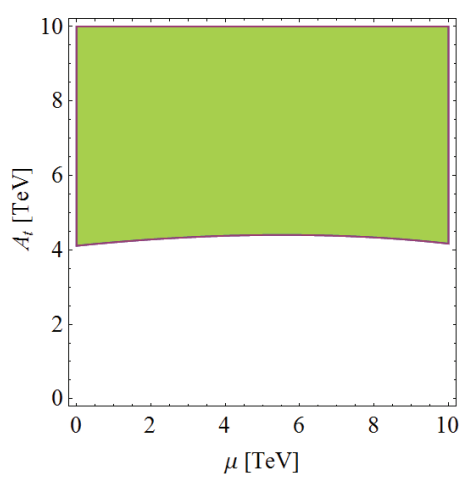

(c)

Figure 2. The allowed regions defined by Eq. (12) for $m_{h}=125 \mathrm{GeV}$ and (a) $-\tan \beta=1, M_{S}=1.5 \mathrm{TeV}$, (b) $\tan \beta=10, M_{S}=1.5 \mathrm{TeV}$ and (c) $\tan \beta=10, M_{S}=5 \mathrm{TeV}$. Yellow and green areas correspond to an allowed parameter range for the Higgs potential $U=U^{(2)}+U^{(4)}+U^{(6)}$; yellow area corresponds to an allowed parameter range for the pottential $U^{(2)}+U^{(4)}$ in the one-loop expansion of the effective Higgs potential.

For example if $\kappa_{i}=0$, then

$$
\begin{aligned}
\Delta & =-\left[-2 \operatorname{Re} \mu_{12}^{2}+v^{2}\left(3 \lambda_{6} c_{\beta}^{2}+3 \lambda_{7} c_{\beta}^{2}+\lambda_{345} s_{2 \beta}\right)\right]^{2} \\
& -\left[v^{2}\left(-\lambda_{7}+3 \lambda_{6} \cot ^{2} \beta+4 \lambda_{1} \cot ^{3} \beta\right)+2 \operatorname{Re} \mu_{12}^{2} \csc ^{2} \beta\right] \\
& \times\left[v^{2}\left(-4 \lambda_{2}-3 \lambda_{7} \cot \beta+\lambda_{6} \cot ^{3} \beta\right)-2 \operatorname{Re} \mu_{12}^{2} \cot \beta \csc ^{2} \beta\right] s_{\beta}^{4} \tan \beta>0,
\end{aligned}
$$

where $\operatorname{Re} \mu_{12}^{2}$ is defined by eq. (7). Allowed domains on the plane $\left(\mu, A_{t}\right)$ for fixed values of $M_{S}$ and $\tan \beta$ are shown in figure 2. They are reconstructed for the case when the lightest CP-even scalar mass $m_{h}=125 \mathrm{GeV}$ and masses of other Higgs bosons are positively defined. Several examples of the mass spectrum of scalars can be found in [9]. The combination of green and yellow regions includes an allowed $A_{t}$ and $\mu$ range if the Higgs potential terms $U^{(2)}+U^{(4)}$ are extended by $U^{(6)}$ operators, eq. (5), and the yellow area represents the allowed region for $U^{(2)}+U^{(4)}$ terms only. Visible deviations of the contours for the dimension-four potential are observed if the values of $A_{t}$ respect the conditions of eq. (11).

Configuration for $U^{(2)}+U^{(4)}+U^{(6)}$ in the case of "good" $\left(A_{t}, \mu\right)$ parameters for the effective potential decomposition is shown in figure 3 (a). The potential has two minima and is positively defined at a large $\phi_{i}^{0}$. So, in this case, the vacuum in which we live is true and stable. But if $A_{t}$ and $\mu$ are chosen from the yellow area of figure 2 (a), two minima degenerate to a gully which forms a saddle configuration in the origin, see figure 3 (b). The conditions defined by eq. (12) are not respected in this case and corrections coming from dimension-six operators spoil the vacuum stability. In the case $\left\|U_{i j}\right\|=0$, the gully forms a flat direction when the stationary point at the origin is degenerate and nonisolated [14].

For the LHC analyses the MSSM multidimensional parameter space is reduced to specific benchmark parameter sets which demonstrate different collider phenomenology. Six benchmark sets which are specified in [8] are usually denominated as $m_{h}^{\max }, m_{h}^{\bmod +}, m_{h}^{\text {mod- }}$, light stop, light stau and $\tau$-phobic scenarios. In these benchmark scenarios $M_{S}<1.5 \mathrm{TeV}, \mu<M_{S}$ (except $\tau$-phobic scenario) and $A_{t}=X_{t}+\mu / \tan \beta$, where $X_{t} \sim O(1) M_{S}$, so large values of $A_{t}$ where the difference between various radiative corrections is appreciable can be achieved only for extremely small $\tan \beta$ region (see eq. 11 ), where one can expect an evidence of additional contributions. The minimum of the potential 


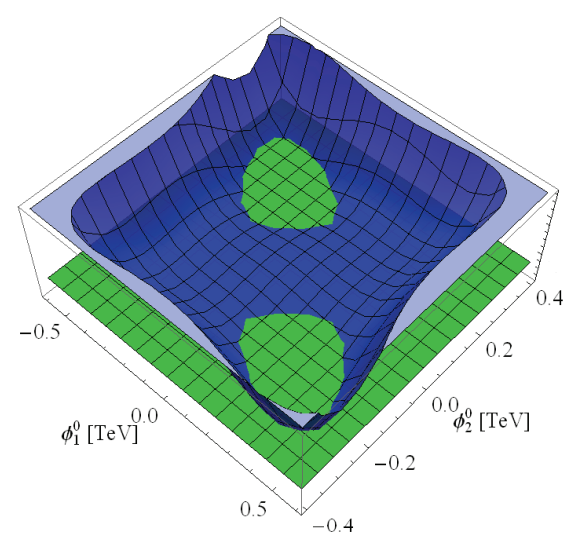

(a)

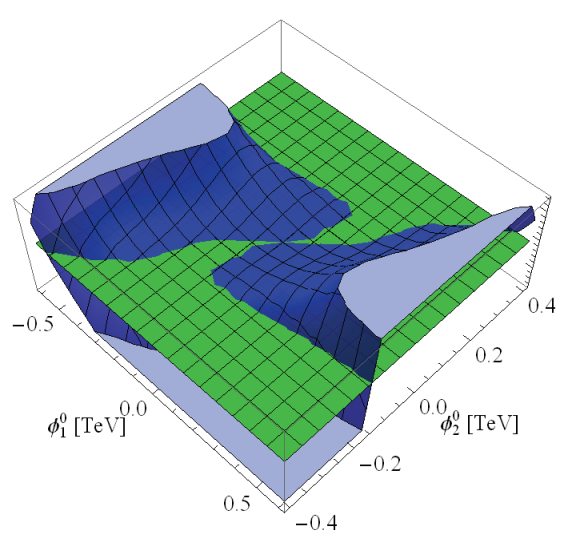

(b)

Figure 3. Higgs potential forms, see (1), for $M_{S}=1.5 \mathrm{TeV}, \tan \beta=1$ and (a) $-A_{t}=\mu=1.5 \mathrm{TeV}$, (b) $-A_{t}=7.5 \mathrm{TeV}$, $\mu=2 \mathrm{TeV}$, see also figure 2 (a). The plane $U=0$ is shown in green colour.

disappears only at low $\tan \beta$ for light stop and $\tau$-phobic scenarios while other demonstrate remarkable stability with respect to radiative corrections.

\section{Summary}

The problem of the SM vacuum stability looks less alarming in the MSSM extension. The best enquiry would be if the beyond SM parameters can be chosen in such a way that the vacuum would be always stable. In the case of two-doublet Higgs sector, the potential has two identical minima, one of them is a vacuum in which we live. The conditions defined by eq. (12) guarantee the stable vacuum, but modification of Higgs potential demonstrates that some parameter sets respecting the minimization in the dimension-four version are not acceptable for the dimension-six extension. Instead of the stable minimum with vev's one can have a saddle configuration at the origin. New terms of dimensionsix in the fields are significant if eq. (11) are respected. In the majority of the MSSM benchmark scenarios the local minimum is stable with respect to dimension-six terms, but for scenarios light stop and $\tau$-phobic the low $\tan \beta$ region is excluded.

The authors are grateful to I. Volobuev for useful remarks. This work was supported by Grant No. NSh7989.2016.2.

\section{References}

[1] G. Aad et al. (ATLAS Collaboration), Phys. Lett. B 716, 1(2012); S. Chatrchyan et al. (CMS Collaboration), Phys. Lett. B 716, 30(2012)

[2] Aad G. et al. (ATLAS and CMS Collaborations), JHEP 1608, 045 (2016); Aad G. et al. (ATLAS and CMS Collaborations), Phys. Rev. Lett. 114, 191803 (2015)

[3] F. Bezrukov, M. Kalmykov, B. Kniehl, and M. Shaposhnikov, JHEP 1210, 140 (2012); J. EliasMiro et al, Phys. Lett. B 709, 222 (2012); J. Casas, J. Espinosa, and M. Quiros, Phys. Lett. B 382, 374 (1996); A. Linde, Phys. Lett. B 92, 209 (1980); H. D. Politzer and S. Wolfram, Phys. Lett. B 82, 242 (1979); ibid, B 83421 (1979); N. V. Krasnikov, Yad. Fiz., 28, 549 (1978); L. Maiani, G. Parisi, and R. Petronzio, Nucl. Phys. B 136, 115 (1978) 
[4] A. Djouadi, Phys. Rep. 459, 1 (2008); H. Haber and G. Kane, Phys. Rep. 117, 75 (1985)

[5] S. Coleman and E. Weinberg, Phys. Rev. D 7, 1888 (1973)

[6] M. Carena, J. R. Espinosa, M. Quiros, and C. E. M. Wagner, Phys. Lett. B 355, 209 (1995)

[7] H. E. Haber and R. Hempfling, Phys. Rev. D 48, 4280 (1993)

[8] M. Carena, S. Heinemeyer, O. Stal, C. E. M. Wagner, and G. Weiglein, Eur. Phys. J. C 732552 (2013)

[9] M. N. Dubinin and E. Yu. Petrova, Phys. Rev. D 95, 055021 (2017)

[10] S. Kanemura and K. Yagyu, Phys. Lett. B 751, 289 (2015)

[11] K. Inoue, A. Kakuto, H. Komatsu, and S. Takeshita, Prog. Theor. Phys. 68, 927 (1982); R. A. Flores and M. Sher, Ann. Phys. (N.Y.) 148, 95 (1983)

[12] E. Akhmetzyanova, M. Dolgopolov, and M. Dubinin, Phys. Rev. D 71, 075008 (2005); Phys. Part. Nucl. 37, 677 (2006)

[13] S. Y. Choi, M. Drees, and J. S. Lee, Phys. Lett. B 48157 (2000)

M. Carena, J. Ellis, A. Pilaftsis, and C. E. M. Wagner, Nucl. Phys. B 586, 92 (2000)

[14] M. N. Dubinin and E. Yu. Petrova, Theor. Math. Phys. 184, 1170 (2015) 